\title{
Folate Receptor Autoimmunity and Cerebral Folate Deficiency in Low-Functioning Autism with Neurological Deficits
}

Authors

Affiliations

\author{
V. T. Ramaekers ${ }^{1}$, N. Blau' ${ }^{2}$, J. M. Sequeira ${ }^{3}$, M.-C. Nassogne ${ }^{4}$, E. V. Quadros ${ }^{3}$
}

Division of Child Neurology, University Hospital Aachen, Aachen, Germany

Division of Chemistry and Biochemistry, University Children's Hospital Zürich, Zürich, Switzerland

Department of Medicine/Cell Biology, SUNY-Downstate Medical Center, Brooklyn, NY, USA

Department of Child Neurology, Université Catholique de Louvain, Louvain, Belgium
Key words

- low-IQ autism

- Kanner syndrome

- neurodevelopmental delay

- cerebral folate deficiency

- folate receptor

autoimmunity received $\quad 02.06 .2007$

accepted $\quad 05.03 .2008$

\section{Bibliography}

DOI $10.1055 / \mathrm{s}-2008-1065354$

Neuropediatrics 2008;

39: 1-6

(c) Georg Thieme Verlag KG

Stuttgart · New York

ISSN 0174-304X

Correspondence

Dr. V. T. Ramaekers, MD, PhD

Division of Child Neurology

University Hospital Aachen

Pauwelsstraße 30

52074 Aachen

Germany

Tel.: +49/241/808 8652

vramaekers@skynet.be

\section{Abstract}

$\nabla$

Reduced folate transport to the CNS was identified in two autism spectrum disorders, i.e., Rett syndrome and infantile low-functioning autism with neurological abnormalities. Twenty-five patients with early-onset low-functioning autism with or without neurological deficits, were evaluated for serum folate, cerebrospinal fluid (CSF) 5-methyltetrahydrofolate (5MTHF), and serum FR autoantibodies of the blocking type to determine the significance of folate receptor (FR) autoantibodies with respect to folate transport across the blood-CSF barrier. In spite of normal serum folate, CSF 5MTHF was low in 23 of 25

\section{Introduction}

\section{$\nabla$}

Autism is defined as a behavioural syndrome of early childhood, which can be divided into five clinical subtypes, i.e., early infantile low-functioning autism (Kanner syndrome), high-functioning autism (Asperger syndrome), disintegrative disorder (Heller syndrome), Rett syndrome and atypical autism (PDD-NOS) [5,9]. These autism syndromes share impaired functions involving sociability, language, non-verbal communicative skills, and imagination, together with narrow, rigid, and perseverative interests and activities. Autism is regularly associated with neurological deficits including stereotypy, difficulties with posture, gait and balance, hypotonia, toe walking, clumsiness, dyspraxia, as well as disorders of sleep and attention, language disorders and cognitive deficits. By adulthood a third of the individuals with the autistic spectrum disorder will have had at least two unprovoked epileptic seizures $[1,6,16]$. Autism with no evident cause, so-called primary autism, represents the major group, whereas secondary autism is responsible for $10-0 \%$ of all cases. The general patients. The reduced CSF folate in 19 of these 23 patients could be explained by serum FR autoantibodies blocking the folate binding site of the membrane-attached FR on the choroid epithelial cells. Oral folinic acid supplements led to normal CSF 5MTHF and partial or complete clinical recovery after 12 months. Serum FR autoimmunity appears to represent an important factor in the pathogenesis of reduced folate transport to the nervous system among children with earlyonset low-functioning autism associated with or without neurological deficits. Early detection of FR autoantibodies may be a key factor in the prevention and therapeutic intervention among this subgroup of patients with autism.

belief is that primary autism is caused by the impact of unidentified environmental factors in a genetically predisposed child [12].

We have previously reported that folate transport to the CNS is reduced in a number of children suffering from two autism spectrum disorders, i.e., Rett syndrome and infantile-onset low-functioning autism [13-5]. Among 28 patients affected by the infantile-onset cerebral folate deficiency (CFD) syndrome, 5 patients were found to manifest low-functioning autism with neurological deficits [15]. In 4 of these 5 autistic patients with low 5MTHF in their cerebrospinal fluid (CSF), the aetiology was attributed to circulating autoantibodies in their serum directed against the folate receptors (FR). In patients with CFD these FR autoantibodies bind to the FR expressed on the choroid plexus and block the folate binding site, thereby inhibiting the transport of folate into the CSF [15].

Thus, FR autoimmunity and CFD appear to be common factors that play a crucial role in the cause and pathogenesis of autism spectrum disorders or in a particular subgroup of the autism spectrum with co-existing neurological deficits. 
The objective of the present study was to determine if children with early-onset low-functioning autism with or without neurological deficits have co-existing CFD and FR autoimmunity.

\section{Patients and Methods \\ V \\ Patient population}

The 25 patients selected for this study had early-onset low-functioning autism associated with one or more of the major features of the infantile-onset CFD syndrome: 1) marked unrest, irritability and insomnia during the first year of life; 2) deceleration of head growth between 4 and 18 months; 3) psychomotor retardation followed by developmental arrest with or without regression; 4) hypotonia and ataxia presenting during the first two years; 5) pyramidal deficits starting in the lower limbs; 6) dyskinesias (choreoathetosis, ballism); and 7) epilepsy [13,15]. The diagnosis of infantile-onset low-functioning autism was established on the basis of the DSM-IV criteria with further assessment using the Autism Diagnostic Observation Schedule (ADOS) in conjunction with the Autism Diagnostic Interview (ADI) and the Vineland scales for children around 3 years $[10,11,18]$.

In all children we ruled out infections during pregnancy, birth and neonatal injuries, hearing deficits, chromosomal disorders, defects of intracellular signalling as measured by abnormal platelet function, genetic abnormalities and inborn errors of metabolism, conditions known to be associated with secondary autism $[12,16]$.

All parents provided written informed consent for participation after the study had been approved by the University Hospital Aachen ethics committee.

\section{Serum and CSF folate analysis}

In all patients CSF was obtained according to a standardized protocol between 8:30 and 10:00 a.m. for analysis of 5-methyltetrahydrofolate, as described previously [8]. The CSF results were compared with previously described values in 100 age-matched controls $[2,4]$. Serum folic acid, vitamin B12, homocysteine, and amino acids were determined in all patients. The serum folate was compared with the values obtained from age-matched healthy controls.

\section{Serum and CSF assay of FR autoantibodies}

For each patient and an age-matched healthy control, serum was collected for assay of FR autoantibodies of the blocking type. All identifying information was removed from the serum specimens and they were identified only after the assays were completed and the results forwarded for analysis.

The procedure for identifying autoantibodies against FRs has been described previously [17]16). In brief, the patient's serum was acidified, charcoal treated, neutralized and incubated overnight with FR, purified from human placental membranes, followed by adding $\left[{ }^{3} \mathrm{H}\right]$ folic acid. Blocking autoantibodies, if present, prevent the binding of $\left[{ }^{3} \mathrm{H}\right]$ folic acid that binds to the receptors in a 1:1 molar ratio. The amount of radioactivity bound to the receptors is inversely proportional to the titre of the blocking autoantibodies and is expressed as picomoles of $\left[{ }^{3} \mathrm{H}\right]$ folic acid blocked from binding to the receptor per $\mathrm{mL}$ of the serum. Available stored CSF samples were also assayed for blocking FR autoantibodies.

\section{Analysis of FR1 and FR2 genes}

The FR1 gene maps to chromosome 11q13.3-q13. 5 and is composed of seven exons spanning $6.7 \mathrm{~kb}$, whereas the FR2 gene is comprised of 5 exons and maps to the same chromosome locus at a distance of $23 \mathrm{~kb}$ from the FR1gene. PCR amplification of FR1 and FR2 genes was performed on DNA isolated from whole blood using gene specific primers spanning intron-exon boundaries. The PCR products of $\sim 250 \mathrm{bp}$ each, were isolated and sequenced [7].

\section{Treatment protocol}

In patients with low 5MTHF levels in CSF, treatment consisted of oral supplementation with folinic acid starting at a dose of $1.0 \mathrm{mg} / \mathrm{kg} /$ day $[13,15]$. Follow-up studies during treatment with folinic acid included repeated clinical assessment and EEG recordings. Clinical follow-up after folinic acid treatment included re-evaluation of the DSM-IV criteria, including the triad of social impairment, impaired communication and restricted behaviour with perseverative interests and activities. A lumbar puncture for measurement of 5MTHF was performed after 3-6 months following start of treatment. If CSF 5MTHF did not normalize, the dose of folinic acid was increased up to 2$3 \mathrm{mg} / \mathrm{kg} /$ day, divided in two doses. All studies and investigations were performed after informed parental consent was obtained.

\section{Results}

$\nabla$

\section{Patient characteristics}

Selection criteria for the twenty-five patients included early-onset low-functioning autism associated with at least one or more of the following seven major clinical criteria (see - Table 1) encountered in the infantile-onset CFD syndrome, i.e.: 1) marked unrest, irritability and insomnia in 22 patients; 2) Deceleration of head growth in 11 patients; 3) psychomotor retardation and developmental arrest in 23 patients with regression in 1 patient, and cognitive decline in 1 patient (vide infra); 2 patients (patients 1,18 ) with Kanner syndrome had normal motor development; 4) hypotonia and ataxia in 20 patients; 5) distal pyramidal tract signs of the lower limbs in 9 patients; 6 ) dyskinesias in 9 patients (choreoathetosis in 5 and ballism in 4 patients); and 7) epilepsy in 11 patients.

Four patients fulfilled the criteria of infantile low-functioning autism (Kanner syndrome; patients 1, 18, 22, 24) associated with unrest, irritability and insomnia. Their history disclosed delayed acquisition of motor milestones in 2 of 4 patients (patients 22, 24), and repeated neurological examination was normal. In twenty-one patients three or more of the above-mentioned major criteria were present and in two of these patients regression and cognitive decline occurred. In one patient (patient 12) the regression of mental and motor functions occurred after the age of 3.5 years leading to a severe neurological state manifesting all major criteria of CFD. The other patient (patient 21) developed normally with mild speech delay until the age of 26 months when she developed acute lymphocytic leukaemia. A few months after starting chemotherapy, including methotrexate, she manifested cognitive decline and developed autism followed later by choreoathetosis, ataxia, and seizures.

\section{Serum and CSF folate}

Routine blood counts, serum vitamin B12, homocysteine and amino acids were within the normal range in all patients. Serum 


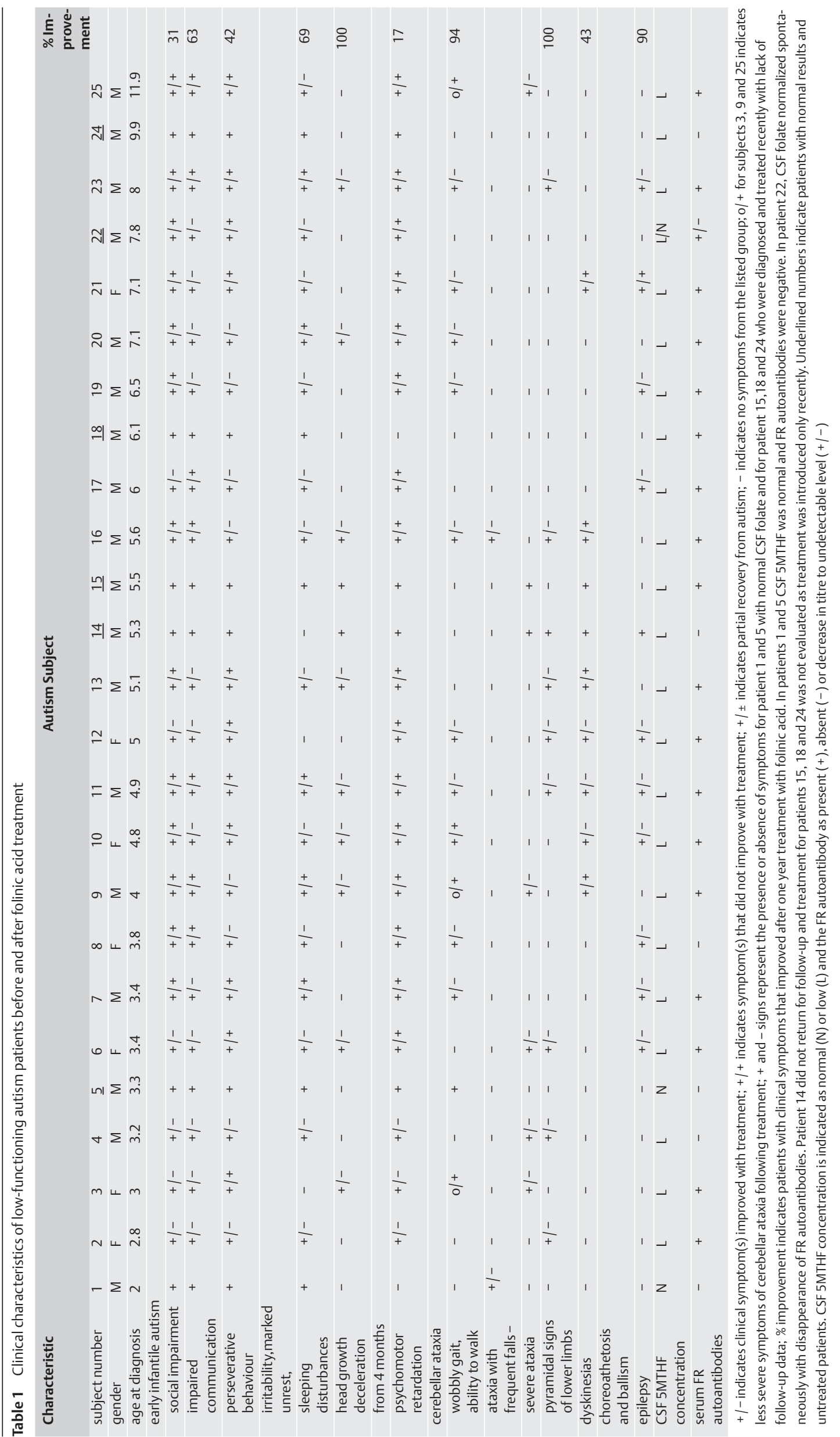


Table 2 Patient characteristics, laboratory values and statistical analysis

\begin{tabular}{|c|c|c|c|c|}
\hline Analyte & Autism & Controls & Statistical Test & Significance \\
\hline \multicolumn{5}{|c|}{ patient characteristics } \\
\hline male:female ratio & $18: 7$ & $14: 11$ & Fisher test & NS, $p=0.1886$ \\
\hline \multicolumn{5}{|l|}{ age (years) } \\
\hline - median & 6.88 & 6.76 & $\mathrm{t}$-value $=0.96$ & NS \\
\hline - range & $2.8-12.3$ & $3.3-11.4$ & & \\
\hline \multicolumn{5}{|l|}{ laboratory values } \\
\hline \multicolumn{5}{|c|}{ serum FR autoantibodies (pmoles/mL serum) } \\
\hline - number positive & 19 & 0 & Fisher test & $p<0.0001$ \\
\hline - mean Titre & 1.05 & 0 & & \\
\hline - range & $0.1-4.19$ & & & \\
\hline \multicolumn{5}{|l|}{ serum Folate (nM) } \\
\hline- mean & 25.62 & 25.2 & $\mathrm{t}$-value $=0.76$ & NS \\
\hline - range & $8.8-47$ & $8.3-45$ & & \\
\hline \multicolumn{5}{|l|}{$5 \mathrm{MTHF}$ in CSF (nM) } \\
\hline \multicolumn{5}{|l|}{ before treatment } \\
\hline- mean & 27.3 & 82 & t-value $=7.77$ & $P<0.001$ \\
\hline - range & $0-85.6$ & $44-181$ & & \\
\hline \multicolumn{5}{|c|}{ after folinic acid treatment } \\
\hline- mean & 75.46 & 82 & $\mathrm{t}$-value $=0.75$ & NS \\
\hline - range & $44.7-175$ & $44-181$ & & \\
\hline
\end{tabular}

folate levels in patients were normal and did not show statistical differences when compared to healthy controls ( 0 Table 2).

The mean CSF concentration of 5MTHF was significantly decreased in all 25 patients with autism associated with or without neurological deficits ( $27.3 \mathrm{nmol} / \mathrm{L}$ compared to $82 \mathrm{nmol} / \mathrm{L}$ for healthy age-matched controls; see 0 Table 2 ).

Two patients had normal CSF folate values; one patient with macrocephaly (patient 1) fulfilled all the criteria of infantile low-functioning autism (Kanner syndrome), while the other patient with normal head growth (patient 5) suffered from lowfunctioning autism, marked motor delay and gait ataxia. Except for these two patients with normal folate values in their CSF, 23 patients had CSF folate values well below the lowest value of the reference range of age-matched controls. Before folinic acid substitution, a follow-up lumbar puncture in 6 patients (patients 7 , $9,16,17,22,23$ ) with low CSF folate showed a further decline of CSF 5MTHF values.

\section{Autoantibodies against folate receptors}

At the time of testing for serum autoantibodies against the folate receptor (FR) the mean age was 6.88. years (range from 2.8 to 12.3 years). There were no statistical differences for age and gender distribution between the patients and healthy controls (see - Table 2). The human FR antigen used in the autoantibody testing was purified from human placental membranes. The two patients with normal CSF folate values, namely the patient with macrocephaly and Kanner syndrome (patient 1) and the patient with autism, motor delay and ataxia (patient 5), had no blocking FR autoantibodies. Four out of 23 patients (1 patient with Kanner syndrome, i.e., patient 24; and 3 patients with low-IQ autism and neurological deficits, i.e., patients $4,8,14$ ), who had low CSF folate levels, did not have FR autoantibodies in their serum. The results showed that 19 out of the 23 patients with low CSF 5MTHF had autoantibodies of the blocking type against the human FR with a mean value of $1.05 \mathrm{pmol}$ FR blocked $/ \mathrm{mL}$ serum (range: 0.1-4.19). All 19 patients with low CSF folate and positive FR autoantibodies suffered from low-functioning autism and neurological deficits, except for the two patients with Kanner

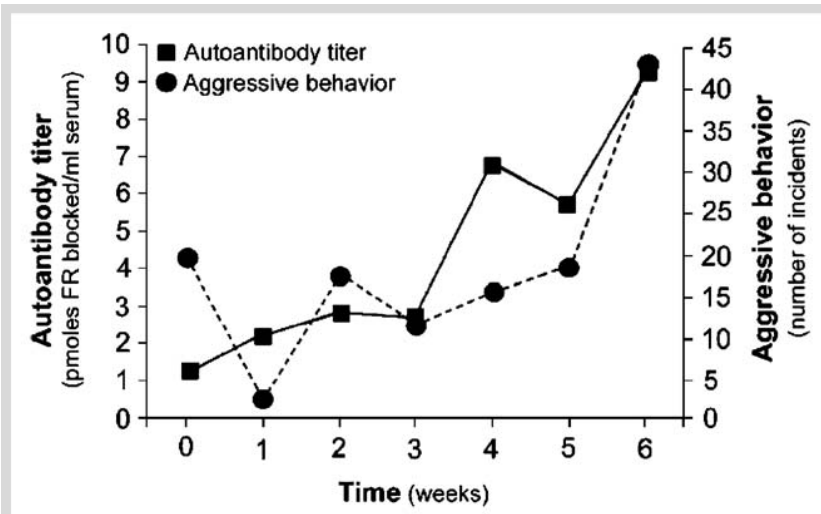

Fig. 1 Changes in antibody titre and behavioural incidents over a period of six weeks in an 8.5-year-old girl with autism and neurological deficits (patient 21). The FR autoantibody titre rose from 1.2 to a maximum of 9.2 pmoles FR blocked $/ \mathrm{mL}$ serum during this period (-口-). The increase in autoantibody titre was followed by increased incidents of aggressive behaviour (-・-).

syndrome who were neurologically normal. All age-matched controls tested negative for FR autoantibodies.

In the patient with cognitive decline (patient 21), autism and signs of CFD followed a few months after chemotherapy for acute lymphocytic leukaemia, which included the antifolate drug methotrexate. The parents noticed cyclic phases during periods of 6 weeks after completion of chemotherapy, where aggression, loss of communicative skills and seizures alternated with phases where these signs were less manifest. During this period, weekly collection of serum for 6 weeks showed a nearly five-fold rise of FR autoantibodies from 1.2 up to a maximum titre at $9.2 \mathrm{pmol}$ FR receptor blocked/mL serum. The increase in antibody titre was followed two weeks later by more incidences of aggressive behaviour, decreased communicative skills and more seizures at the time of maximum FR autoantibody levels (๑ Fig. 1). 


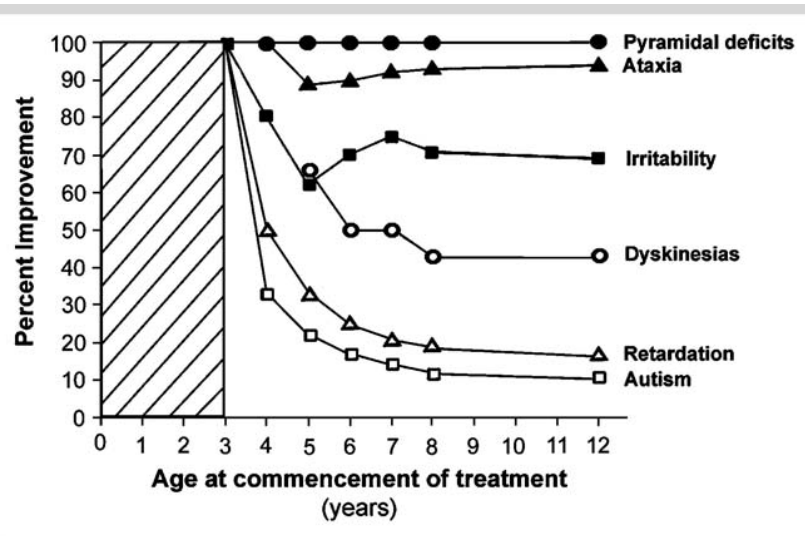

Fig. 2 Response to therapy as indicated by improvement in neurological symptoms plotted against age at initial presentation and commencement of treatment. Following folinic acid administration for at least one year, a decrease in overall response to therapy with increasing age was observed. Improvement of mental retardation (- $\triangle$-), autism (- $\square-$ ), dyskinesias (-O-) and irritability (--) declines progressively if diagnosis and treatment are started at later ages, while pyramidal deficits (- $\bullet-)$, ataxia $(-\boldsymbol{\Delta}-)$ show a better response throughout and are less affected by age. The hatched area represents the age at which treatment can reverse or substantially improve all of the neurological symptoms.

Serum FR autoantibodies determined on two occasions in 5 other patients also showed minor or major titre fluctuations. In one patient with Kanner autism (patient 22), who had marked CSF folate fluctuations with subnormal values over the years, the low titre of FR autoantibodies had turned negative on follow-up, coinciding with normalization of CSF folate levels. One other patient (patient 17) who initially had no FR autoantibodies, developed autoantibodies with a titre of $3.18 \mathrm{pmol}$ FR blocked/ $\mathrm{mL}$ serum after 9 months.

In 5 patients with positive serum FR autoantibodies, where a stored CSF sample was available, FR autoantibodies were not detected in CSF.

\section{Genetic analysis of FR1 and FR2}

The coding exons of FR1 and FR2 genes showed normal sequences indicating that the low CSF folate could not be attributed to genetic errors of these genes.

\section{Treatment and outcome}

Two patients with low CSF folate received no treatment because of spontaneous disappearance of FR autoantibodies with normalization of CSF folate in one (patient 22), and the other did not return for follow-up (patient 14).

Twenty-one patients with low CSF folate received treatment with high doses of $\mathrm{N}^{5}$-formyltetrahydrofolate (calcium folinate) at a dose of $1-3 \mathrm{mg} / \mathrm{kg} /$ day. The complete follow-up data for 3 (patients 15, 18, 24) of the 21 patients who started treatment only recently, are not available yet and have not been included in the present study. In all 18 patients treated with calcium folinate, CSF analysis showed normalization of 5MTHF levels ( $\bullet$ Table 1 ). Two patients (patients 2,4) who were diagnosed early and received treatment were cured with full recovery from autism and neurological deficits. In the whole group these two patients were among the youngest and were detected at 2 years 8 months and at 3 years and 2 months. Three older patients (patients 11,23, 25), diagnosed and treated from the age of $4.9,8$ and 11.9 years, did not recover from autism but showed improve- ment of their neurological deficits. The remaining thirteen patients in the age range of three and seven years showed a good response after treatment with improvement of most neurological deficits, but only partial recovery from their autism. The partial recovery in the latter group of 13 patients consisted of amelioration of social impairment in 4 of 13 patients, reversal of impaired communication in 9 of 13 patients and disappearance of perseverative behaviour and restricted interests in 6 of 13 patients. The percentage of overall improvement and improvement of each specific feature plotted against the age at diagnosis and prior to treatment ( $\bullet$ Fig. 2) showed a trend towards better outcome if the diagnosis and treatment are established at an earlier age. Although these are preliminary data, the curves also show that outcome of low-functioning autism with neurological deficits will be excellent if diagnosis is established before the age of three years. It also shows that treatment provided in this study has a positive effect on the alleviation of epilepsy, pyramidal deficits and ataxia, but shows less response on irritability and a lack of effect on dyskinesias, mental retardation and autism with advancing age.

\section{Discussion}

$\nabla$

Most patients of this study were selected on the basis of earlyonset low functioning autism associated with neurological deficits, while four patients did not manifest neurological deficits and could be classified as having Kanner syndrome. The inclusion of neurological criteria was based upon previous findings in the infantile-onset CFD syndrome where 28 children with low CSF folate fulfilled at least 3 or more of 7 major neurological criteria $[13,15]$. Five of these 28 patients with CFD were found to have low-functioning autism associated with neurological deficits. In 4 of these 5 autistic children, FR autoantibodies were present in the serum, explaining the low CSF folate due to reduced folate transport across the choroid plexus [15].

With the exception of two, all 23 patients with low-functioning autism of the present study had low CSF folate accompanied by the presence of serum autoantibodies directed against FR in 19 patients. Testing of CSF in 5 patients with positive serum FR autoantibodies, showed the absence of FR autoantibodies in their CSF, indicating that there is no intrathecal production or transfer of FR autoantibodies from blood.

The phenotype of most patients consisted of a combination of low-functioning autism and neurological deficits. However, three out of the four patients with the Kanner syndrome, also had low CSF folate and FR autoantibodies were present in two of these patients. In this context it should be stressed that within the classification of autism spectrum disorders the presence or absence of neurological deficits is often not mentioned or disregarded, particularly in the group of low-functioning infantile autism and atypical autism (PDD-NOS). Therefore, it remains unclear how frequently FR autoimmunity and CFD occur in autism without neurological deficits compared to autism associated with neurological abnormalities. For future studies, documentation and reporting of neurological abnormalities among patients with autism is imperative in order to correlate CFD and FR autoimmunity with either group.

Interestingly, repeated determination of serum FR autoantibodies in one patient showed marked fluctuations in the titre that directly correlated with the severity of the clinical disease. This lends further support to the existence of an inverse correlation 
between the titre of FR autoantibodies with the transport and availability of folate to the brain. Fluctuations and the appearance or disappearance of FR autoantibodies were also observed in 5 other patients.

Characterization of serum FR autoantibodies demonstrated the blocking properties of these autoantibodies with respect to folate binding by the human FR. A number of genetically determined factors are important in early programming and maintenance of the immune system to avoid responses to the body's own tissues and antigenic determinants [3]. It may well be that in autism one or more genetic factors involved could disrupt tolerance against one's own antigens leading to autoimmunity and interfere with the normal growth and developmental processes of the nervous system $[12,16]$. However, genetic factors that predispose to FR autoimmunity may provide a reasonable explanation for the clinical phenotype as two of the youngest diagnosed and treated patients were cured from their autism following treatment with folinic acid. Screening of serum for FR autoantibodies should be performed among infants and children manifesting autistic features and developmental delay, because early identification and intervention for these disorders appears to improve the prognosis and outcome.

\section{References}

1 Ahsgren I, Baldwin I, Goetzinger-Falk C, Erikson A, Flodmark O, Gillberg C. Ataxia, autism, and the cerebellum: a clinical study of 32 individuals with congenital ataxia. Dev Med Child Neurol 2005; 47: 193-198

2 Blau N, Bonafe L, Blaskovics ME. Disorders of phenylalanine and tetrahydrobiopterin metabolism. In: Blau N, Duran M, Blaskovics ME, Gibson KM (Eds.), Physician's guide to the laboratory diagnosis of metabolic diseases. Berlin: Springer 2003; 96

3 Bowcock AM, Lovett M. Zeroing in on tolerance. Nat Med 2001; 3: 279-281

4 Curtius HC, Blau N, Kuster T. Pterins. In: Hommes FC (Ed.), Techniques in Diagnostic Human Biochemical Genetics. New York: Wiley-Liss $1991 ; 377-396$
5 Gillberg C, Coleman M. The Biology of the Autistic Syndromes 3rd edn., Mac Keith Press: Lonodn 2000

6 Haas RH, Townsend J, Courchesne E, Lincoln AJ, Schreibman L, YeungCourchesne R. Neurologic abnormalities in infantile autism. J Child Neurol 1996; 11: 84-92

7 Heil SG, Put NMJ Van der, Trijbels FJM, Gabreels FJ, Blom HJ. Molecular genetic analysis of human folate receptors in neural tube defects. Eur J Hum Genet 1999; 7: 393-396

8 Hyland $K$, Surtees $R$. Measurement of 5-methyltetrahydrofolate in cerebrospinal fluid using HPLC with coulometric electrochemical detection. Pteridines 1992; 3: 149-150

9 Kanner L. Autistic disturbances of affective contact. Nervous Child 1943; 2: 217-250

10 Le Couteur A, Rutter M, Lord C, Rios P, Robertson S, Holdgrafer M, MacLennan J. Autism diagnostic interview: a standardized investigator-based instrument. J Autism Develop Disord 1989; 19: 363-387

11 Lord C, Rutter M, Goode S, Heemsbergen J, Jordan J, Mawhood L, Schopler E. Autism diagnostic observation schedule: a standardized observation of communicative and social behavior. J Autism Develop Disord 1989; 19: 185-212

12 Muhle R, Trentacoste SV, Rapin I. The genetics of autism. Pediatrics 2004; 113: e472-486

13 Ramaekers V, Blau N. Cerebral folate deficiency. Dev Med Child Neurol 2004; 46: 843-851

14 Ramaekers V, Hansen SI, Holm J, Opladen T, Senderek J, Hausler M, Heimann G, Fowler B, Maiwald R, Blau N. Reduced folate transport to the CNS in female Rett patients. Neurology 2003; 61: 506-514

15 Ramaekers VT, Rothenberg SP, Sequeira JM, Opladen T, Blau $N$, Quadros EV. Autoantibodies to folate receptors in the cerebral folate deficiency syndrome. N Engl J Med 2005; 352: 1985-1991

16 Rapin I, Katzman R. Neurobiology of autism. Ann Neurol 1998; 43: 7-14

17 Rothenberg SP, da Costa MP, Sequeira JM, Bracco J, Roberts JL, Weedon J. Quadros EV. Autoantibodies against folate receptors in women with a pregnancy complicated by a neural-tube defect. $\mathrm{N}$ Engl J Med 2004; 350: 134-142

18 Stone WL, Ousley OY, Hepburn SL, Hogan KL, Brown CS. Patterns of adaptive behavior in very young children with autism. Am J Ment Retard 1999; 104: 187-199 